\title{
INFLUÊNCIA DO TIPO DE FERMENTO NAS CARACTERÍSTICAS FÍSICO-QUÍMICAS DE QUEIJO MINAS ARTESANAL DO SERRO - MINAS GERAIS, MATURADO EM CONDIÇÕES CONTROLADAS
}

\section{Influence of the type of ferment in the physicochemical characteristics of cheese Minas artisanal do Serro - Minas Gerais, matured in controlled conditions}

\begin{abstract}
Rosana Campos do Vale ${ }^{1 *}$, Maria Paula Jensen Rodrigues ${ }^{1}$, José Manoel Martins ${ }^{1}$
\end{abstract}
\section{RESUMO}

Na produção do queijo Minas artesanal do Serro são utilizados leite cru, coalho e fermento lático natural, designado de "pingo". Alguns produtores da região do Serro, fazem uso da "rala", parte ralada do próprio queijo artesanal, com 3 a 5 dias de fabricação, que é adicionada ao leite em substituição ao "pingo". Sabendo-se que condições controladas de umidade relativa do ar (UR) e temperatura também podem influenciar as características dos queijos durante a maturação. O objetivo deste trabalho foi avaliar a influência do tipo de fermento ("pingo" ou "rala") nas características físico-químicas do queijo Minas artesanal do Serro, maturado em condições de UR e temperatura controladas. Queijos artesanais de seis produtores da região do Serro, cadastrados no Instituto Mineiro de Agropecuária, sendo três fabricados com "pingo" e três com "rala", foram maturados sem embalagens, em câmara de maturação com UR média de $85 \%$ e temperatura de $20{ }^{\circ} \mathrm{C}$, durante 31 dias na Cooperativa dos Produtores Rurais do Serro. As análises foram realizadas nos tempos 3, 10, 17, 24 e 31 dias de maturação. Para os parâmetros de umidade, acidez, gordura, gordura no extrato seco, cinzas, cloreto de sódio, sal na umidade e índices de extensão de maturação não se observou diferença $(p>0,05)$ entre os queijos fabricados com "pingo" e "rala". As altas temperaturas de maturação e de umidade relativa do ar mostraram não serem condições adequadas de maturação para

1 Instituto Federal do Sudeste de Minas Gerais, Campus Rio Pomba, Av. Dr. José Sebastião da Paixão, Lindo Vale, 36180-000, Rio Pomba, MG, Brasil. E-mail: rosanacampos17@yahoo.com.br

* Autor para correspondência. 
os queijos artesanais do Serro, já que modificaram as características físico-químicas e de aparência do produto.

Palavras-chave: fermento endógeno; maturação; queijo artesanal.

\begin{abstract}
In the production of the Minas Gerais artisanal cheese from Serro, raw milk, rennet and natural lactic culture are used, called "pingo". Some producers in the Serro region make use of the "rala", a grated part of the artisanal cheese itself, with 3-5 days of manufacture, which is added to milk instead of "pingo". The objective of this work was to evaluate the influence of the type of culture ("pingo". or "rala") on the physicochemical characteristics of the Minas Gerais artisanal cheese from Serro, matured under controlled temperature and relative humidity $(\mathrm{RH})$ conditions. Artisanal cheeses from six producers from the Serro region, registered at the Instituto Mineiro de Agropecuária (IMA), three made with "pingo" and three with "rala", were matured without packaging in a maturation chamber with an average $\mathrm{RH}$ of $85 \%$ and a temperature of $20{ }^{\circ} \mathrm{C}$ for 31 days at the Cooperativa dos Productores Rurais do Serro. The analyzes were performed at times 3, 10, 17, 24 and 31 days of maturation. For the parameters of moisture content, acidity, fat content, fat in the dry extract, ash, sodium chloride, salt in the humidity and indices of extension of maturation, there was no difference $(p>0.05)$ between cheeses made with "pingo" and "rala". The high temperatures and relative air humidity used in the maturation were not suitable for the Serro artisanal cheese maturation, since they modified the physical and chemical characteristics of the product.
\end{abstract}

Keywords: endogenous culture; maturation; artisanal cheese.

\section{INTRODUÇÃO}

O queijo Minas artesanal (QMA) do Serro é um produto de extrema importância não só para o estado de Minas Gerais, mas também para o Brasil, tanto pela geração de renda e ocupação, quanto por sua conotação sociocultural, sendo produzido quase que exclusivamente pela agricultura familiar (BRANT et al., 2007).

No processo de produção do QMA do Serro são utilizados leite cru e fermento lático natural, popularmente designado por "pingo". Nos últimos anos, alguns produtores de queijo artesanal da região têm utilizado como fermento lático o próprio queijo artesanal, com três a cinco dias de fabricação, que é ralado e adicionado ao leite, sendo denominado pelos produtores como "rala" (FIGUEIREDO, 2014).
Não existe ainda uma regulamentação para a utilização da "rala" na produção de queijos artesanais. Faltam estudos científicos que indiquem quais os efeitos desse tipo de fermento nas características físico-químicas e microbiológicas dos queijos artesanais, uma vez que alguns produtores e consumidores afirmam, empiricamente, que há diferença entre os tipos de fermento ("pingo" e "rala").

Além do tipo de fermento, outro fator que pode afetar as características dos queijos é a condição de maturação a que são submetidos. Esta por sua vez é influenciada por dois elementos fundamentais: temperatura e umidade relativa do ar (UR), que podem interferir tanto no crescimento microbiano, quanto na composição do queijo.

Em Minas Gerais, foram criados dois Centros de Qualidade do QMA, nas cidades de 
Medeiros (região da Serra da Canastra) e Rio Paranaíba (região do Cerrado), visando maior padronização do processo de maturação e fortalecimento das Associações de Produtores para a venda coletiva dos queijos (EMATER, 2013).

Estes Centros permitem, por meio de um acordo entre o Ministério da Agricultura e o serviço de inspeção sanitária de Minas Gerais, que os queijos sejam comercializados para as demais unidades da Federação, num processo equivalente ao Sistema Brasileiro de Inspeção (SISBI). A Portaria $n^{0} 1.305$, de 30 de abril de 2013, permite que o produto saia dos Centros de Qualidade diretamente para a mesa do consumidor brasileiro (IMA, 2013).

A Cooperativa dos Produtores Rurais do Serro (COOPSERRO), motivada em conhecer melhor os efeitos da maturação dos queijos artesanais, agregar valor aos mesmos e favorecer sua comercialização, construiu em suas instalações uma câmara de maturação para os queijos artesanais, com temperatura e UR controladas, gerando condições de experimentação do processo de maturação.

O QMA, embora em sua maior parte seja consumido fresco, tradicionalmente foi reconhecido pela segurança e sabor diferenciado promovido pelo tempo de maturação (MARTINS, 2015). Para os queijos do Serro, este tempo estabelecido pela Portaria $\mathrm{n}^{\mathrm{o}} 1.305$ (IMA, 2013) é de 17 dias, para queijarias integrantes do Sistema Brasileiro de Inspeção de Produtos de Origem Animal (SISBI/ POA), baseado em Martins (2006), que estudou queijos artesanais desta região num período em que nenhuma queijaria ainda havia se cadastrado junto ao Instituto Mineiro de Agropecuária (IMA).

Ainda não se sabe qual a influência da maturação dos QMAs em condições controladas (temperatura e UR) e nem o tempo de maturação que podem levar para que os mesmos sejam colocados no mercado atendendo à legislação (IMA, 2013), além da influência dos tipos de fermentos na qualidade dos produtos.

Desta forma, verifica-se a necessidade de estudos científicos que permitem avaliar os efeitos da maturação de queijos artesanais em condições de temperatura e UR controladas, nas características e na qualidade deste produto.

Diante disso, o presente trabalho teve como objetivo avaliar a influência do tipo de fermento nas características físico-químicas do queijo Minas artesanal do Serro, durante o período de maturação em condições controladas.

\section{MATERIAL E MÉTODOS}

Queijos artesanais de 6 produtores rurais da região do Serro, que comercializam na Cooperativa, devidamente cadastrados no Instituto Mineiro de Agropecuária (IMA), sendo três (3) fabricados com pingo e três (3) com rala, foram maturados em câmara de maturação em condições controladas durante 31 dias (outubro de 2017) na Cooperativa dos Produtores Rurais do Serro.

Esses queijos ficaram sem embalagens, em prateleiras de madeira, em câmara de maturação controlada com umidade relativa do ar (UR) média de $85 \%$ e temperatura de $20{ }^{\circ} \mathrm{C}$. A cada dois dias os queijos foram lavados para limpeza de suas superfícies.

Respeitando-se os tempos de análises, 3, 10, 17, 24 e 31 dias de maturação, os queijos artesanais foram coletados diretamente na câmara de maturação da Cooperativa dos Produtores Rurais do Serro, devidamente embalados, a vácuo, e acondicionados em caixas de isopor com material refrigerante, para serem transportados até o Laboratório de Físico-Química do Departamento de Ciência e Tecnologia de Alimentos do Instituto Federal de Ciência e Tecnologia do Sudeste de Minas Gerais (IF Sudeste MG), Campus Rio Pomba, para a realização das análises. Uma amostra 
de cada produtor, em cada tempo, foi analisada quanto às características físico-químicas.

Para a determinação da umidade, cinzas, gordura, cloretos, acidez titulável, $\mathrm{pH}$ e gordura no extrato seco (GES), foram utilizados os métodos oficiais para as referidas análises, descritos na Instrução Normativa $\mathrm{n}^{\mathrm{o}} 68$, de 12 de dezembro de 2006 (BRASIL, 2006). Para a determinação de proteína, índice de extensão de maturação (IEM) e índice de profundidade de maturação (IPM), foram utilizados os métodos oficiais, descritos na Instrução Normativa $n^{\circ} 68$ (BRASIL, 2006) e Pereira et al., (2001).

A relação sal/umidade dos queijos foi calculada de acordo com a equação 1 (PAULA et al., 2009):

$\%$ de sal na umidade $=(\%$ Cloretos $\mathrm{x} 100) /$ $(\%$ Cloretos $+\% \mathrm{U})$

Sendo que:

$\%$ de sal na umidade do queijo $(\mathrm{m} / \mathrm{m})$; $\%$ Cloretos $=$ teor de cloreto de sódio no queijo $(\mathrm{m} / \mathrm{m})$;

$\% \mathrm{U}=$ teor de umidade do queijo $(\mathrm{m} / \mathrm{m})$.

Foram realizados dois tratamentos $(\mathrm{T} 1$ = queijo fabricado com "pingo" e T2 = queijo fabricado com "rala"), com amostras de 3 (três) produtores diferentes por tratamento, escolhidos aleatoriamente, no mês de outubro de 2017.

O experimento foi implementado sob o delineamento inteiramente casualizado (DIC), uma vez que, cada produtor realizou apenas um tratamento, não sendo possível isolar o efeito dos produtores, considerando blocos. Foi considerado o esquema fatorial $2 \times 5$, sendo os fatores tipo de fermento ("pingo e rala") e período de maturação (3, $10,17,24$ e 31 dias). Os dados analisados foram submetidos à análise de variância com o objetivo de verificar os efeitos principais e das interações entre os fatores estudados.
Além da análise de variância, foi realizado também o teste Shapiro-Wilk para testar a normalidade, o teste do Durbin-Watson para verificar a independência e, por fim, o teste de Breusch-Pagan para avaliar a homogeneidade de variâncias. Além disso, devido a falta de modelos que se ajustasse adequadamente, foi utilizado o teste de Tukey para avaliar a diferença entre as médias considerando a interação dos fatores tratamento (tipo de fermento) e tempo (período de maturação em dias). Em todas as análises considerou-se um nível de significância de 5\% para as conclusões acerca dos resultados obtidos. Todas as análises estatísticas foram feitas com o auxílio do software "R" (R Core team, 2017).

\section{RESULTADOS E DISCUSSÃO}

Não houve diferença $(\mathrm{p}>0,05)$ entre os QMA da região do Serro fabricados com "pingo" e "rala" para a maioria dos aspectos físico-químicos avaliados (Tabela 1), com exceção de $\mathrm{pH}$ para o tempo 3 dias $(\mathrm{p}<0,05)$.

Somente acidez, cinzas, cloreto de sódio e a relação sal na umidade (Tabela 1) não sofreram influência do tempo de maturação $(\mathrm{p}>0,05)$, ao passo que os demais aspectos, em, pelo menos, um dos tempos de análise variaram seus valores $(\mathrm{p}<0,05)$.

A determinação do teor de umidade de QMA é muito importante, pois está relacionada com as contagens microbiológicas. A umidade dos queijos fabricados com "pingo" foi maior para os tempos 3 e 10 dias de maturação, quando comparada com os tempos 24 e 31 dias $(p<0,05)$. Já os queijos fabricados com "rala" apresentaram maior conteúdo de umidade nas duas primeiras semanas em relação à última semana de maturação $(\mathrm{p}<0,05)$. 
Tabela 1 - Valores médios dos parâmetros físico-químicos de queijos Minas artesanal do Serro fabricados com "pingo" e com "rala", maturados em condições controladas

\begin{tabular}{|c|c|c|c|c|c|c|c|}
\hline \multirow[b]{2}{*}{ Análises } & \multirow{2}{*}{$\begin{array}{c}\text { Tipo } \\
\text { de } \\
\text { fermento }\end{array}$} & \multicolumn{6}{|c|}{ Período de maturação (dias) } \\
\hline & & 3 & 10 & 17 & 24 & 31 & $\begin{array}{c}\text { Médias } \\
\text { Trat. }\end{array}$ \\
\hline \multirow{2}{*}{$\begin{array}{c}\text { Umidade } \\
(\%)\end{array}$} & pingo & $56,24^{\mathrm{Ab}}$ & $56,74^{\mathrm{Ab}}$ & $51,04^{\mathrm{Aab}}$ & $47,29^{\mathrm{Aa}}$ & $44,97^{\mathrm{Aa}}$ & $51,25^{\mathrm{a}}$ \\
\hline & rala & $54,79^{\mathrm{Ac}}$ & $53,10^{\mathrm{Abc}}$ & $50,44^{\text {Aabc }}$ & $46,54^{\mathrm{Aab}}$ & $44,95^{\mathrm{Aa}}$ & $49,96^{\mathrm{a}}$ \\
\hline \multirow{2}{*}{$\begin{array}{l}\text { Acidez (\% } \\
\text { ácido lático) }\end{array}$} & pingo & $1,74^{\mathrm{Aa}}$ & $1,97^{\mathrm{Aa}}$ & $1,35^{\mathrm{Aa}}$ & $1,41^{\mathrm{Aa}}$ & $1,22^{\mathrm{Aa}}$ & $1,53^{\mathrm{a}}$ \\
\hline & rala & $1,24^{\mathrm{Aa}}$ & $1,98^{\mathrm{Aa}}$ & $1,18^{\mathrm{Aa}}$ & $1,22^{\mathrm{Aa}}$ & $1,24^{\mathrm{Aa}}$ & $1,37^{\mathrm{a}}$ \\
\hline \multirow[t]{2}{*}{$\mathrm{pH}$} & pingo & $4,75^{\mathrm{Aa}}$ & $4,80^{\mathrm{Aa}}$ & $5,16^{\mathrm{Aab}}$ & $5,37^{\mathrm{Ab}}$ & $5,36^{\mathrm{Ab}}$ & $5,09^{\mathrm{a}}$ \\
\hline & rala & $5,14^{\mathrm{Bab}}$ & $4,92^{\mathrm{Aa}}$ & $5,17^{\text {Aab }}$ & $5,47^{\mathrm{Ab}}$ & $5,64^{\mathrm{Ab}}$ & $5,27^{\mathrm{b}}$ \\
\hline \multirow{2}{*}{$\begin{array}{c}\text { Gordura } \\
(\%)\end{array}$} & pingo & $20,17^{\mathrm{Aa}}$ & $22,75^{\mathrm{Aab}}$ & $22,92^{\mathrm{Aab}}$ & $26,25^{\mathrm{Aac}}$ & $29,33^{\mathrm{Ac}}$ & $24,28^{a}$ \\
\hline & rala & $20,75^{\mathrm{Aa}}$ & $22,50^{\mathrm{Aab}}$ & $22,17^{\text {Aab }}$ & $26,25^{\mathrm{Ab}}$ & $25,92^{\text {Aab }}$ & $23,51^{\mathrm{a}}$ \\
\hline \multirow{2}{*}{$\begin{array}{l}\text { GES } \\
(\%)\end{array}$} & pingo & $45,97^{\mathrm{Aa}}$ & $53,43^{\mathrm{Aab}}$ & $47,03^{\mathrm{Aa}}$ & $49,82^{\mathrm{Aab}}$ & $53,30^{\mathrm{Ab}}$ & $52,08^{a}$ \\
\hline & rala & $45,96^{\mathrm{Aa}}$ & $48,53^{\mathrm{Aa}}$ & $44,73^{\mathrm{Aa}}$ & $49,04^{\mathrm{Aa}}$ & $47,08^{\mathrm{Aa}}$ & $49,58^{\mathrm{a}}$ \\
\hline \multirow{2}{*}{$\begin{array}{c}\text { Cinzas } \\
(\%)\end{array}$} & pingo & $3,11^{\mathrm{Aa}}$ & $3,15^{\text {Аa }}$ & $2,84^{\mathrm{Aa}}$ & $4,05^{\mathrm{Aa}}$ & $3,05^{\mathrm{Aa}}$ & $3,24^{\mathrm{a}}$ \\
\hline & rala & $3,83^{\mathrm{Aa}}$ & $3,46^{\mathrm{Aa}}$ & $3,55^{\mathrm{Aa}}$ & $4,36^{\mathrm{Aa}}$ & $3,49^{\mathrm{Aa}}$ & $3,74^{\mathrm{a}}$ \\
\hline \multirow{2}{*}{$\begin{array}{l}\mathrm{NaCl} \\
(\%)\end{array}$} & pingo & $1,38^{\mathrm{Aa}}$ & $1,47^{\mathrm{Aa}}$ & $1,19^{\mathrm{Aa}}$ & $1,42^{\mathrm{Aa}}$ & $1,63^{\mathrm{Aa}}$ & $1,41^{\mathrm{a}}$ \\
\hline & rala & $1,74^{\mathrm{Aa}}$ & $1,58^{\mathrm{Aa}}$ & $1,85^{\mathrm{Aa}}$ & $1,73^{\mathrm{Aa}}$ & $1,82^{\mathrm{Aa}}$ & $1,74^{\mathrm{a}}$ \\
\hline \multirow{2}{*}{$\begin{array}{c}\text { Sal/Umidade } \\
(\%)\end{array}$} & pingo & $2,39^{\mathrm{Aa}}$ & $2,57^{\mathrm{Aa}}$ & $2,30^{\mathrm{Aa}}$ & $2,91^{\mathrm{Aa}}$ & $2,93^{\mathrm{Aa}}$ & $2,62^{\mathrm{a}}$ \\
\hline & rala & $3,10^{\mathrm{Aa}}$ & $2,87^{\mathrm{Aa}}$ & $3,52^{\mathrm{Aa}}$ & $3,57^{\mathrm{Aa}}$ & $3,17^{\mathrm{Aa}}$ & $3,24^{\mathrm{a}}$ \\
\hline \multirow{2}{*}{$\begin{array}{c}\text { Proteína } \\
(\%) \\
\end{array}$} & pingo & $17,76^{\mathrm{Aa}}$ & $19,74^{\mathrm{Ab}}$ & $20,65^{\mathrm{Ab}}$ & $21,19^{\mathrm{Ab}}$ & $21.42^{\mathrm{Ab}}$ & $20,64^{\mathrm{a}}$ \\
\hline & rala & $17,75^{\mathrm{Aa}}$ & $21,22^{\mathrm{Aab}}$ & $21,52^{\mathrm{Abc}}$ & $21,29^{\mathrm{Aab}}$ & $24,40^{\mathrm{Bc}}$ & $20,74^{\mathrm{a}}$ \\
\hline \multirow{2}{*}{$\begin{array}{l}\text { IEM } \\
(\%)\end{array}$} & pingo & $15,42^{\mathrm{Aa}}$ & $24,43^{\mathrm{Aa}}$ & $20,40^{\mathrm{Aa}}$ & $27,27^{\mathrm{Aa}}$ & $27,53^{\mathrm{Aa}}$ & $23,01^{\mathrm{a}}$ \\
\hline & rala & $16,47^{\mathrm{Aa}}$ & $19,60^{\mathrm{Aa}}$ & $18,17^{\mathrm{Aa}}$ & $20,26^{\mathrm{Aa}}$ & $23,50^{\mathrm{Aa}}$ & $19,60^{\mathrm{a}}$ \\
\hline \multirow{2}{*}{$\begin{array}{l}\text { IPM } \\
(\%)\end{array}$} & pingo & $10,19^{\mathrm{Aa}}$ & $11,98^{\mathrm{Aa}}$ & $15,83^{\mathrm{Aab}}$ & $22,68^{\mathrm{Ab}}$ & $22,06^{\mathrm{Ab}}$ & $16,55^{\mathrm{a}}$ \\
\hline & rala & $9,32^{\mathrm{Aa}}$ & $13,88^{\mathrm{Aab}}$ & $13,42^{\mathrm{Aab}}$ & $19,73^{\mathrm{Ab}}$ & $19,78^{\mathrm{Ab}}$ & $15,23^{a}$ \\
\hline
\end{tabular}

Médias seguidas pela mesma letra não diferem entre si, ao nível de 5\% de significância pelo teste de Tukey. As letras minúsculas comparam os períodos de maturação (dias), enquanto as letras maiúsculas comparam os tipos de fermentos.

$\mathrm{NaCl}=$ cloreto de sódio; $\mathrm{GES}=$ gordura no extrato seco; $\mathrm{pH}=$ potencial hidrogeniônico; IEM = índice de extensão de maturação; IPM = índice de profundidade de maturação; Trat. = Tratamentos. 
Ainda em relação à umidade, independentemente do tipo de fermento utilizado, decresceu mais acentuadamente entre a segunda e quarta semana de maturação dos queijos. Ao longo do tempo de maturação o valor médio de umidade aproximou-se de $44,7 \%$ aos 31 dias de maturação.

Vale destacar que neste trabalho os queijos fabricados com ambos os fermentos ("pingo" e "rala") foram classificados como queijos de alta umidade entre os tempos $3 \mathrm{e}$ 24 dias de maturação, passando a queijos de média umidade na última semana de análise (31 dias). A utilização de umidade relativa do ar elevada da câmara de maturação pode ter contribuído para a manutenção do elevado teor de umidade dos queijos nas primeiras semanas de maturação. Assim, os queijos só atenderam às exigências da legislação estadual (MINAS GERAIS, 2008) a partir dos 31 dias de maturação, que preconiza um teor de umidade inferior a $46 \%$.

Brumano (2016), em seu experimento com queijo Minas artesanal do Serro, fabricados com "pingo" e "rala", maturados à temperatura ambiente, verificou que a sua classificação foi diretamente influenciada pelo tempo, sendo de alta umidade quando tinham de 2 a 8 dias de maturação, de média umidade, quando tinham de 15 a 29 dias, e de baixa umidade quando o tempo foi superior a 36 dias de maturação. Já Oliveira et al. (2013), ao analisarem queijos artesanais da mesma região obtiveram um teor médio de umidade de $47,83 \%$, sendo classificados como queijos de alta umidade.

Em relação à acidez titulável não foi observada diferença $(p>0,05)$ entre os queijos fabricados com "pingo" e "rala" e nem influência do período de maturação para nenhum dos dois tratamentos $(\mathrm{p}>0,05)$ (Tabela 1). A média geral da acidez encontrada nesse trabalho foi de $1,45 \%$ de ácido lático.

A acidez é um importante aspecto composicional de queijos, pois influencia o cres- cimento de microrganismos e a atividade enzimática ao longo da maturação (LAVASANI et al., 2011). A acidez proveniente da produção do ácido lático a partir da degradação da lactose pelas bactérias afeta de maneira direta o $\mathrm{pH}$ e expulsão de soro da massa durante a fabricação e início da fase de cura (SOUSA et al., 2014). Ferraz (2016) encontrou valores de acidez de queijos artesanais da Serra da Canastra, maturados em Centros de Qualidade, variando de 0,53 a 1,01, valores bem inferiores aos obtidos neste estudo.

Para o $\mathrm{pH}$, o queijo fabricado com "pingo" apresentou valores menores $(\mathrm{p}<0,05)$ que os queijos fabricados com "rala", mas somente no tempo 3 dias de maturação. A partir do $24^{\circ}$ dia de maturação é que os queijos fabricados com "pingo" apresentaram aumento de $\mathrm{pH}(\mathrm{p}<0,05)$. Já os queijos fabricados com "rala" apresentaram menor $\mathrm{pH}$ para o tempo 10 dias de maturação ( $\mathrm{p}<0,05)$, quando comparado aos tempos 24 e 31 dias.

Essa variação nos valores de $\mathrm{pH}$ pode ter ocorrido devido a degradação proteica proveniente da atividade de enzimas coagulantes, enzimas naturalmente presentes no leite (plasmina), enzimas de bactérias láticas não starters (NSLAB) ou enzimas de bactérias láticas presentes no soro fermento (pingo) como também na "rala". Essa atividade resulta na formação de compostos nitrogenados alcalinos, contribuindo para o aumento de $\mathrm{pH}$ (DORES et al. 2013; MARTINS et al., 2015).

Para o teor de proteína não houve diferença $(p>0,05)$ entre os queijos fabricados com "pingo" e "rala" nos tempos 3, 10, 17 e 24 dias de maturação. Entretanto constatou-se diferença $(\mathrm{p}<0,05)$ no tempo 31 dias de maturação. Oliveira et al. (2013), ao analisarem queijos artesanais da região do Serro obtiveram um teor de proteínas de $14,08 \%$, valor bem inferior ao obtido no presente trabalho.

Há de se considerar que as condições de maturação dos QMA do Serro, no presente 
trabalho, podem ter influenciado diretamente os valores de proteína encontrados, com variações significativas $(\mathrm{p}<0,05)$ para os dois tratamentos, mas abaixo daqueles encontrados por outros pesquisadores (MARTINS, 2015; FERRAZ, 2016).

Os demais compostos nitrogenados, índice de extensão de maturação (IEM) e índice de profundidade de maturação (IPM), também não apresentaram diferença entre os tratamentos $(\mathrm{p}>0,05)$ em nenhum dia avaliado (Tabela 1).

O IEM, medido pela relação percentual de nitrogênio solúvel em pH4,6/nitrogênio total, é exercido, na sua maior parte, por influência do coalho. Já o IPM é mensurado pela relação percentual nitrogênio solúvel em ácido tricloroacético 12\%/nitrogênio total, sendo influenciado principalmente pelas enzimas do fermento utilizado (COSTA JÚNIOR et al., 2014).

Para o conteúdo de gordura dos queijos não se constatou diferença $(p>0,05)$, para os dois tipos de fermentos utilizados (Tabela 1). O percentual de gordura encontrado nas amostras produzidas na região do Serro são semelhantes aos valores obtidos por Machado et al. (2004), que encontraram em QMA do Serro teor lipídico na ordem de 29,22\%.

A gordura é o componente que naturalmente sofre maior variação no leite, sendo influenciada pela estação do ano, alimentação animal, estágio de lactação e raça dos animais (FERRAZ, 2016), somado ao fato do leite utilizado na fabricação de QMA não sofrer nenhum tipo de padronização.

Não houve diferença $(\mathrm{p}>0,05)$ entre os QMA da região do Serro fabricados com "pingo" e "rala" para gordura no extrato seco (GES) (Tabela 1). Foi observado apenas, para os queijos fabricados com o "pingo", que os resultados das 3 primeiras semanas foram menores que aqueles encontrados em 31 dias de maturação $(\mathrm{p}<0,05)$.

A legislação brasileira (BRASIL, 1996) classifica os queijos de acordo com o seu conteúdo de gordura no extrato seco (GES) em desnatados (<10,0\%), magros (10 a $24,9 \%$ ), semi gordos (25,0 a 44,9\%), gordos $(45,0$ a $59,9 \%)$ e extra gordos ou duplo creme $(\geq 60,0 \%)$. Dessa forma, tanto os queijos fabricados com "pingo" e "rala" se classificaram como queijos gordos, com exceção daqueles fabricados com rala, no tempo 17 dias, que foram classificados como semigordos (Tabela 1).

Para cinzas, cloreto de sódio e sal na umidade (Tabela 1), não houve diferença $(p>0,05)$ entre os queijos fabricados com "pingo" e "rala" e nem influência do período de maturação para nenhum dos dois tratamentos $(\mathrm{p}>0,05)$.

Oliveira et al. (2017), obtiveram em seu estudo com queijos artesanais, uma média de teor de cloretos de $3,75 \%$, sendo que no seu estudo, os valores de cloreto de sódio ficaram acima do valor de $2 \%$ (permitido pela legislação) durante todo o período de maturação.

Segundo Martins (2006), o tipo de salga a seco, utilizada pelos queijeiros da região do Serro, não possibilita uma padronização adequada do teor de sal no queijo, podendo haver variações.

O teor médio de sal na umidade obtido para os queijos fabricados com "pingo" foi de $2,62 \%$ e para amostras de queijos fabricadas com "rala" foi de 3,24\%. Ferraz (2016), ao analisar queijos artesanais fabricados com "pingo" da Serra da Canastra, obteve para a relação sal na umidade, um teor de 2,94\% para queijos maturados no Centro de Qualidade e 3,23\% para os queijos maturados nas próprias queijarias. De acordo com Paula et al. (2009), as enzimas lipases e proteases são mais ativas em teores variando de 0,5 a $2,5 \%$ de sal na umidade. Valores maiores que $5 \%$ favorecem a solubilização da caseína durante o processo de maturação, devido a trocas entre íons de cálcio e sódio. 


\section{CONCLUSÕES}

O único aspecto físico-químico que apresentou diferença entre os fermentos endógenos, "pingo" e "rala", utilizados na fabricação dos queijos Minas artesanais da região do Serro, foi $\mathrm{pH}$.

As condições de temperatura e de umidade relativa do ar durante a maturação de queijos Minas artesanais do Serro precisam ser mais bem estudadas, pois estas podem influenciar as características sensoriais e microbiológicas deste produto.

\section{AGRADECIMENTOS}

À Cooperativa dos Produtores Rurais do Serro (COOPSERRO), em especial ao funcionário Edivaldo da Costa Júnior e ao seu Presidente, Carlos da Silveira Dumond, pela parceria e pelo apoio financeiro para a realização do projeto.

À Fundação de Amparo à Pesquisa do Estado de Minas Gerais (FAPEMIG), pelo apoio financeiro ao projeto.

Ao Instituto Federal de Educação, Ciência e Tecnologia do Sudeste de Minas Gerais, Campus Rio Pomba.

\section{REFERÊNCIAS}

BRANT, L. M. F.; FONSECA, L. M.; SILVA, M. C. C. Avaliação da qualidade microbiológica do queijo-de-minas artesanal do Serro - MG. Arquivo Brasileiro de Medicina Veterinária e Zootecnia, v. 59, n. 6, p. 15701574, 2007.

BRASIL. Ministério da Agricultura, Pecuária e Abastecimento. Instrução Normativa $\mathrm{n}^{\circ} 68$, de 12 de dezembro de 2006. Oficializa os métodos analíticos oficiais físico-químicos, para controle de leite e produtos lácteos. Diário Oficial da República Federativa do Brasil, Brasília, 14 dez. 2006. Seção 1, p. 8.
BRASIL. Portaria $n^{\circ}$ 146, de 07 de março de 1996. Ministério da Agricultura do Abastecimento e da Reforma Agrária. Secretaria Nacional de Inspeção de Produtos de Origem Animal. Regulamentos Técnicos de Identidade e Qualidade dos Produtos Lácteos. Diário Oficial da República Federativa do Brasil, Brasília, 11 de março de 1996. Seção 1, Página 3977.

BRUMANO, E. C. D. C. Impacto do tipo de fermento endógeno na qualidade e tempo de maturação de queijo Minas artesanal produzido em propriedades cadastradas pelo IMA (Instituto Mineiro de Agropecuária) na região do Serro - MG. 2016. 136 f. Tese de Doutorado - Universidade Federal de Viçosa, Viçosa, 2016.

COSTA JÚNIOR, L. C. G. et al. Maturação do queijo Minas artesanal da microrregião Campo das Vertentes e os efeitos dos períodos seco e chuvoso. Revista do Instituto Laticínios Cândido Tostes, v. 69, n. 2, p. 111120, 2014.

DORES, M. T.; NÓBREGA, J. E.; FERREIRA, C. L. L. F. Room temperature aging to guarantee microbiological safety of Brazilian artisan Canastra cheese. Food Science and Technology, v. 33, n. 1, p. 180-185, 2013.

EMATER. Empresa de Assistência Técnica e Extensão Rural do Estado de Minas Gerais. Belo Horizonte, 2013. Medeiros inaugura o primeiro centro de qualidade do queijo Minas artesanal e será o primeiro município a vender o produto fora de Minas Gerais. Disponível em: <http://www.emater.mg.gov. br/portal.cgi?flagweb=site_tpl_paginas internas \& id=11287\#. WEnAB 5 XruP8>. Acesso em: 10 abr. 2016.

FERRAZ, W. M. Queijo Minas artesanal da Serra da Canastra: influência do ambiente 
sobre a maturação. 2016. 93 f. Dissertação (Mestrado em Ciência e Tecnologia de Alimentos) - Instituto Federal de Educação, Ciência e Tecnologia do Sudeste de Minas Gerais - Campus Rio Pomba, 2016.

FIGUEIREDO, S. P. Características do leite cru e do queijo minas artesanal produzidos na região do Serro, Minas Gerais e, produção de queijos com doces. 2014. 108 f. Dissertação (Mestrado em Zootecnia) - Universidade Federal dos Vales do Jequitinhonha e Mucuri, Diamantina, 2014.

INSTITUTO MINEIRO DE AGROPECUÁRIA (IMA). Portaria no 1305, de 30 de abril de 2013. Diretrizes para a produção do queijo Minas artesanal. Belo Horizonte, 2013.

LAVASANI, A. R. S. et al. Changes in physicochemical and organoleptic properties of traditional Iranian cheese Lighvan during ripening. International Journal of Dairy Technology, v. 65, n. 1, p. 64-70, 2011.

MACHADO E. C.; FERREIRA, C. L. L. F.; FONSECA, L. M. Características físicoquímicas e sensoriais do queijo Minas artesanal produzido na região do Serro, Minas Gerais. Ciência e Tecnologia de Alimentos, v. 24, n. 4 , p. 516-521, 2004.

MARTINS, J. M. et al. Determining the minimum ripening time at room temperature and under refrigeration of Minas artisanal cheese, a traditional Brazilian cheese. Brazilian Journal of Microbiology, v. 46, n. 1, p. 219-230, 2015.

MARTINS, J. M. Características físico-químicas e microbiológicas durante a maturação do queijo Minas artesanal da região do Serro. 2006. 158 f. (Doutorado em Ciência e Tecnologia de Alimentos) - Universidade Federal de Viçosa. Viçosa, 2006.

MINAS GERAIS. Decreto $n^{\circ} 44.864$, de $1^{\circ}$ de agosto de 2008. Altera o Regulamento da Lei $\mathrm{n}^{\circ} 14.185$, de 31 de janeiro de 2002 , que dispõe sobre o processo de produção de queijo Minas artesanal. Minas Gerais, Belo Horizonte, 02 ago. 2008. Diário do executivo, p. 1.

OLIVEIRA, A. L. D. et al. Caracterização do queijo Minas artesanal do cerrado mineiro da região do Alto Paranaíba. The Journal of Engineering and Exact Sciences, v. 3, n. 6, p. 0824-0828, 2017.

OLIVEIRA, D. F. et al. Caracterização físicoquímica de queijos Minas artesanal produzidos em diferentes microrregiões de Minas Gerais. Revista Brasileira de Economia Doméstica, v. 24, n. 2, p. 185-196, 2013.

PAUlA, J. C. J.; CARVAlho, A. F.; FURTADO, M. M. Princípios básicos de fabricação de queijo: do histórico a salga. Revista do Instituto de Laticínios Cândido Tostes, v. 64, n. 367, p. 19-25, 2009.

PEREIRA, D. B. C. et al. Físico-química do leite e derivados: Métodos analíticos. Juiz de Fora: Oficina de Impressão, 2001. 190 p.

SOUSA, A. Z. B. et al. Aspectos físicoquímicos e microbiológicos do queijo tipo coalho comercializados em estados do nordeste do Brasil. Arquivos Instituto Biológico, São Paulo, v. 81, n. 1, p. 30-35, 2014.

TEAM, R. Core. R: A language and environment for statistical computing. Vienna, Austria: R Foundation for Statistical Computing; 2017. 